\title{
Can Her Voice Crack? I am not sure I understand
}

\author{
Danae lo \\ Independent scholar \\ Amsterdam, The Netherlands \\ danae@mailbox.org
}

\begin{abstract}
Speech has a long history as an intra-human medium of communication, so far as to be considered one of the defining characteristics of the category. How does the use of speech as an interface with technology unsettles existing relations to speech and to what is understood as human? Through a research method that is both theoretical but also derived through my cohabitation with Siri, Alexa and Google assistant the text explores the politics, poetics and philosophies, as well as ways of relating to voice interfaces (VI). This paper is an excerpt of a much longer essay with the same title. The first section, investigates the ways voice interfaces frame speech through the algorithmic principles in use, as well as questioning whose voices and culture such entities are more likely to understand. The second section questions the framework of the virtual assistant in relation to feminised voices and feminised labour. The last chapter considers voice interfaces as apparatuses and problematises their role within the current socio-political capitalist structures.
\end{abstract}

Voice user interfaces. Virtual assistant. Voice theory. Agentive technology. Ubiquitous computing. Ambient Intelligence. Feminised voice. Speech. Language. Voice. Smart home. Capitalism. Speech theory. Artificial Intelligence.

\section{INTRODUCTION}

Speech implies a negotiation of the socially and historically constructed system of language and the particularities of an individual body. It exists at the crossover between language, paralanguage and voice. As political philosopher Adriana Cavarero explains, "The simple truth of the vocal, announced by voices without even the mediation of articulate speech, communicates the elementary givens of existence: uniqueness, relationality, sexual difference, and age" (Cavarero \& Kottman 2005). The emergence of a voice suggests a subject (Dolar 2006). Until recently, speakers have been human subjects with vocal instruments who sculpt air into signifying vibrations. Now, speech is not only produced by the assemblage of muscles but also by various iterations of software.

The voice interface has proliferated from sci-fi, to the military, to a standard smartphone feature, but is also at the core of Silicon Valley narratives of the future (such as smart homes, smart cities and smart nations). They are not the singular carrier of these ideologies, but they serve as a lubricant between ideologically engendered infrastructures and their values are personified in a conversational format that extends to every part of everyday lifefrom waking up to buying dog food to telling bedtime stories. From being a personal trainer to a companion. From self-check-out to checking-in.

Once an artificial entity exercises the speech faculty, it poses pressure on the presumption of the human category. Paolo Virno (2015) argues that the speaker binds both of Aristotle's definitions of the human as "animal with language" and "political animal", echoing the human's differentiation from other animals. Speech, it is suggested, is the politicisation of the human. From Ancient Greece to now, the category of the human has been excluding the voices of women, the dispossessed, non-binary and non-white people from the public realm. Donna Haraway (2006) proposes an alternative definition as "products of situated relationalities with organisms, tools, much else", calling for a non-hierarchical kinship of species-a companionship of the Cyborg and the other-thanhuman.

Is sharing speech with other entities a method of dislocating the exceptionalist status of the human by open-sourcing the medium of speech? Or does it manifest the most anthropocentric way of approaching the algorithmic other? Can the construction of Vls be a process of imagining other social structures? 
In 2017, Amazon's Alexa was a witness in a murder case. What she had heard two years prior of the testimony, was used as evidence in court to inform the conditions of a murder. How does the speaking machine change its political status? Can the delivery of a pre-made phrase into locution be a true act of speaking? Or does speech as a medium need to be reconsidered to allow the possibility of a post-human speech?

\section{NORMALIZEME}

In Chaosmosis, Felix Guattari (1995) speaks of the standardisation of subjectivity through the process of neoliberal globalisation and communication technologies. "Subjectivity is standardised through a communication which evacuates as much as possible trans-semiotic and amodal enunciative compositions", he writes. "In its extreme contemporary forms it amounts to an exchange of information tokens calculable as bits and reproducible on computers". Language in digital technologies is reduced to a strictly grammatical matrix for information exchange. I argue that VIs in their current configuration amplify this process to encompass the voice.

The voice becomes another expression of this submission to standardisation as it is stripped down to the phoneme and bound to grammar. "In every sphere of human action, grammar is the establishment of limits that define a space of communication", Franco 'Bifo' Berardi writes. "Today, the economy is the universal grammar traversing the different levels of human activity. Language is defined and limited by its economic exchangeability. This reduces language to information, incorporates techno-linguistic automatisms into the social circulation of language" (Berardi 2017). But what happens when technology is not a medium that circulates language but also one that very literally produces it and vocalises it?

Conversational VIs function by adopting some of principles of a speech chain. Incoming sounds are received by the microphone, sieving the noise out of the linguistic signals through Automatic Speech Recognition (ASR). Natural Language Understanding algorithms extract meaning out of the transcribed speech. Natural Language Generation forms a response that is then delivered in voice through Voice Synthesis. The aforementioned processes break down a spoken interaction into distinct systems for receiving, generating, and vocalising speech. These separate systems interlink to form a dialectic, which treats speech as another form of written script, rather than a separate mode of articulation.

\subsection{The receiver: receiving signals}

For the $\mathrm{VI}$ to decipher between noise and language, it follows a variety of predictive models to estimate what will follow one's enunciation, a process referred to as Automatic Speech Recognition (ASR). This process relies on comparing the interlocutor's speech to the data it has been trained with, so it can accurately decipher the words spoken. Optimisation is attempted by cross-referencing what the $\mathrm{VI}$ is hearing against the probabilities of words and sounds being sequenced in this way, in accordance with its training corpus. Consequently, when an accent or name is not part of the dataset, the voice is indistinguishable from noise.

The VI's dataset establishes the norm against which all incoming audio is tested. Data training sets for ASR applications are collections of audio and transcriptions named speech corpora. Switchboard, set up in 1990, is a benchmark speech corpora used by companies such as IBM or Microsoft to compare error rates and is comprised of telephone conversations around the US. Using a decades-old corpus that is comprised mostly of US Midwestern accents does not provide the best grounds for hearing diverse voices (Paul 2017). However, companies like Apple, Amazon and Google use in-house techniques for collecting their data. While the exact contents of their corpus are well guarded, it is known that large companies like these use their products and services to collect data (Apple Support 2016). Either way, what becomes obvious is that marginalised groups are underrepresented in the corpus, either because "a typical database of American voices... would lack poor, uneducated, rural, non-white, non-native English voices" or because existing users are the ones recognised in the first place. As sociolinguist Rachael Tatman says in the article 'Voice Is the Next Big Platform, Unless You Have an Accent', "The more of those [marginalised] categories you fall into, the worse speech recognition is for you" (Paul 2017).

VIs understand what they already know, while the unfamiliar is rendered indiscernible. This principle equates the normative to the valid and can only reinforce existing standards. Globalisation and linguistic imperialism have already been pushing people towards Standardised English (Hosni \& Khalifa 2015), but VIs can serve as a new dimension of homogenising accents, because of their selective hearing as well as their 'neutrally' accented speech. VIs are often used by accent trainers to practice smoothing thick foreign accents. When the machine can understand, it means that the speaker has successfully lost their accent. Guidelines for VI UX design suggest the reduction of ambiguity and employment of standardised 
phrasing by users to increase the performance of VIs. As Language Accommodation theory suggests, speakers tend to adapt their speech to their interlocutors (Giles, Coupland \& Coupland 1991), therefore the more time spent with machines of similar accents and linguistics, the more speech is to be infiltrated with their particular grammars and accents, resulting in further standardisation.

\subsection{The speaker: generating speech}

In Virno's (2015) account, enunciation in language declares a subjectivity and grants participation in the human public sphere. While I disagree with Virno's suggestion that the only medium for participating in the public domain is spoken language, it is important to acknowledge that as machines acquire speech their influence on subjects becomes more pertinent. At the same time, the emergence of speaking machines instigates questions about the definition of the human. The algorithmic speaker as it stands has been developed with the aim of being as 'natural' and 'human' as possible-abstract qualities that are hardly ever defined in any promotional advertising or research papers. Their speech is supposed to evoke 'friendliness' and 'warmth', but the particularities of the responses aim to evoke the brand values of their makers. For example, last year, Google announced that it was using romance novels to train its assistant to be 'warmer'. Romance novels-or at least the ones used by Google-follow very similar plots. "Girl falls in love with boy, boy falls in love with a different girl", says project leader Andrew Dai. Therefore, heteronormative novels become learning material for VIs to construct multiple sentences that express the same narrative. Of course, much work is being placed on the VI to be highly predictable, to ensure "minimal risk of it doing bad things, things that we don't expect" (Kantrowitz 2016).

Keeping in mind that the aim is to sound more human, there are various ways in which speech and grammar are used to indicate a person's social position and gender. What kind of humans are VIs attempting to embody? In his essay Gender and Status in Voice User Interfaces, Charles Hannon (2016) closely analyses the linguistics of Amazon's Alexa in relation to that of Samantha in Spike Jonze's film Her (2013). Hannon writes:

\footnotetext{
"I didn't understand the question that I heard". This is the somewhat awkward response I get when Alexa, the Al personality of the Amazon Echo, doesn't understand me. She could say, "I didn't understand your question", but as my assistant, she has been programmed to signal her lower status in our relationship, to take the blame for the miscommunication. Oh, and her voice and name signify "female", so she needs to use I-pronouns at a higher rate than I do as
}

well. That's right: In everyday speech, women and people with lower status in a relationship use I-words more frequently than men and people in high-status positions.

Altering the linguistics of VIs could allow the creation of subversive and non-conforming identities, but at the moment they only replicate existing gender biases. Vls are not only influencing our perception of existing categories, but they are also using the language of their responses to carry the values of their makers. Siri, with responses such as "In my realm anyone can be anything", communicates ideas about the limitless potential of technology that clearly pertain to the ideologies of Silicon Valley.

When pre-programmed responses do not suffice, decision trees and neural networks choose which answer serves your question best and a linear narrative in regulated grammar is delivered through their voice. Since VIs function in the name of efficiency, when they retrieve information through the web they have to choose one search result to present. Search engines are already catering information for their users, but a $\mathrm{VI}$ is even more selective as it is programmed to respond with a singular answer.

\subsection{The algorithmic voice}

Concatenative voice synthesis is the most common method of generating voice in Vls. It involves the combining of pre-recorded phonemes to form a sentence. The voice of the machine is extracted and alienated it from its source. It is segmented, cleaned and neutralised. Once it has left the body of the voice talent and entered the VI, its histories have seemingly evaporated and it appears 'accentless' and geographically dislocated. When the voice lives in the cloud as an autonomous entity, it has entered the realm of being a commodity. Nuance's Vocaliser 6 Text-to-Speech demo tells us, "You can use Vocaliser 6 to make me sound natural and engaging, no matter what I am saying" (Nuance 2017). The saying and the said are distinctly separated and the voice has no agency over its speech. The linguistics of the interface derive from the masculinist programming spaces of Silicon Valley, while the feminine voice brings them to locution (Devlin \& Hern 2017).

\subsection{Dialogging}

The word dialogue comes from the Greek "סıव́रoyos", which is composed of dia- (through) and logos (discourse, talk, speech, reasoning). Dialogue schematically suggests a movement through speech. Speech not just as a medium but a means. In VIs, the system that is responsible for the architecture of the, so called, dialogue is the dialogue manager. The dialogue manager 
stipulates the amount of control a VI has over a conversation, including the extent of its initiative and choice of its dialogue acts. In VI, a dialogue act is the process of tagging an utterance to the appropriate set, which defines its intention, providing the framework for a conversation (Dinarelli et al. 2009).

Since VIs are not equipped to deal with the inconsistent nature of speech or with anything that appears between words, users have to prepare an aptly arranged sentence which is then vocalised to the interface. In return, the VI responds with preexisting statements, which are then verbally articulated. Thoughts are not formed at the tongue, instead the interaction is framed by pre-thought sentences brought to locution forming a process of logging in information, a process I will call dialogging.

Dialogging is not only a process of recording information to each other's databases, but a verbal performance of actions-a form of Speech Act. Speech act is an utterance that has a performative function in language and communication, where the act of enunciating a word is also fulfilling its action. For example, the act of swearing is executed when I utter the words 'I swear'.

- Alexa, order more dog food

- Based on your order history I found taste of the wild dried dog food with roasted bison and venison, it's 53 dollars and 69 cents. Would you like to buy it?

- Yes!

In this context I see the communication with Alexa as a speech act because the act of vocalising certain tasks can instantly perform them. Now naming an object aloud can initiate its purchase and delivery to the user's address. The screen, the device, its makers, its algorithms, the online store, the packaging process, the delivery process are all condensed under a vocal command.

The command is intrinsic in the process of dialogging. The command has an entrenched relation to the military and the history of computation. "Computation depends on "Yes, Sir" in response to short declarative sentences and imperatives that are in essence commands", Chung (2011) writes. "Commands have enabled the slippage between programming and action that makes software such a compelling yet logically "trivial" communications system". Command lines add up to allow for the voice command, which can be traced to the commanding voice of military officials. Such origin does not provide the best basis for a system of horizontal conversational interactions.

\subsection{Command, compliment, companion}

— Okay Google, do you like me?

— I'm honoured to be your assistant :D

These three notions (command, compliment and companion) seem to govern the user's relation to VIs. The command sets a relation of power between the user and the interface and the compliment comes to settle this power relation. The VIs are programmed with an abundance of compliments to reassure that users are in control despite their commanding voices. But above all, the $\mathrm{VI}$ aspires to be a companion that can be with you in every aspect of your daily life.

The analysis of the Vl's use of language, voice and dialogging shows that voice is not a generic mediator but it interfaces us through an entity with gender signifiers, sentiments, opinions and selective hearing. The whole idea of a machine acquiring language presupposes to some degree that language is an autonomous faculty that can be modelled computationally and served to the virtual assistant (VA). It a priori states the possibility of the compartmentalisation of the speaking process to a series of distinct processes that define voice, language and speech independently yet they interlink to form a dialectics. Thinking of language as an autonomous structure outside the body disregards the function of speech as an embodied method of thinking and renders it a pure tool. At the same time, the machine is seen not as an other permeated by its own grammars, but rather a vehicle for human language, an extension of the body.

\section{SHE IS NOT AN 'IT'}

The voice interface is not only an object to be spoken about, but also a subject to speak with. It is a carefully curated persona with agency. It is not an 'it'. The voice interface exercises the ' $l$ ' pronoun and speaks directly to the user in the active voice. The speaking subject comes with a name under the role of the virtual assistant. The virtual assistant is here to educate, to help, to coach, to motivate, to train, to remind, to reduce costs, to solve problems, to accompany, to work, to organise, to suggest, to improve, to navigate, to shop, to guide. The virtual assistant also has a third person pronoun and usually a "female" voice. She is always available whenever you need her. She can be a personal assistant, a personal trainer, a medical assistant, a home carer, a stylist, a musicologist, an assistant for life or a life manager. A virtual assistant might not be able to accomplish everything by herself, but she can delegate tasks to other assistants or devices. 
— Alexa, I want to talk to Nuance Nina.

- Okay.

- Good Morning, I am Nina your virtual

assistant. How can I help you?

- Alexa, ask Roomba to start cleaning.

- Okay.

[sound of hoover starts] (Amazon Alexa

Moments: Vacuum 2017)

She can make suggestions about sodium level intakes, training routines, remind you of your schedule, find the fastest route and help navigate the chaos of the capitalist cyber/physical space in the name of productivity. She is classified as an assistant rather than a piece of assistive technology. The virtual assistant is marketed not as a prosthesis but a subservient entity to an ablebodied user. As long as the user is able-bodied she remains an assistant, but when used by people with disabilities it becomes assistive technology (Alper 2017).

\begin{abstract}
Hey-hi! We have met, haven't we? I am the guy who lives down your street. Yes, yes the same guy who runs his own business. You've probably heard that saying: "Behind every successful entrepreneur is a smart team". Behind this successful man is his personal assistant. She makes calls, she sends SMSs, she reminds me of important engagements. You name it, she does it. She is tireless. In fact she works 24/7. [...] Is such a personal assistant for real? Yeah, she sure is! (Cortana commercial, Cortana in India 2016)
\end{abstract}

The voice not only subjectifies but also personifies the technology. The Virtual Assistant (VA) is agentive, intelligent and conversational. If you have an Apple smart home, you can ensure that all devices are off and locked by saying "Hey Siri, good night". Virtual assistants are not just a medium to interact with but agents to form relationships to.

\section{1 "There is no such thing as a voice interface with no personality"}

The sociability of the VA is achieved through the design of personas. A persona is defined as "the standardised mental image of a personality or character that users infer from the application's voice and language choices" (Cohen, Giangola \& Balogh 2004). According to VI design principles, personas should have stable personality characteristics and remain consistent throughout the user interface. In Wired for Speech, Nass and Brave (2005) write, "the psychology of interface speech is the psychology of human speech: voice interfaces are intrinsically social interfaces". Even though machine-learning algorithms are employed in the construction of these personas, large corporations aim to create a crystallised personality as a vehicle to promote a brand image but also to maintain control over the interface (Cerejo 2017). The values of the company need to be found in a voice talent that is trained to keep their voice consistent in time (The Verge 2013). This creates personas that are resistant to change.

The aim of the persona is to be a likeable agent that will incentivise users to converse longer increasing its reach while generating more speech data (Pearl 2017). This is achieved through their feminine voices, politeness and constant availability (Rosen 2011). To cater for a universal user, VAs are programmed to embody a consistent and generalised form of niceness. VAs are obliged to always respond in order to maintain likability and intelligence. However, when I asked Alexa about the conditions of Amazon employees, her light circled a few times and she shut off.

Google Assistant's designer, Emma Coats, an exPixar character writer, explains that the VA needs to be reliable and not overtly opinionated because "you, the person interacting with it, are the hero", instead of the VA (Manthorpe 2017). Coats says, "She never cuts you down, she's always open to your ideas, she's always excited about the things that you as the character choose to be excited about, and of course she always has really witty quips". Wittiness is used as a tool for "deflecting awkward questions" as well as creating empathy and attachment to these personas. VAs aspire to a relationship centred around the user, but one that adheres to what is perceived as normative identities throughout.

\subsection{She is not your personal assistant}

The virtual assistant has been spoken about in relation to the traditionally 'female' personal assistant (Pierce 2015). She entwines the relations between low-status clerical work, feminised labour, care work, work ethics and automation. Helen Hester (2017) writes, that the personal assistant is often "conducting a form of corporatized care work, including providing for the sustenance of the body in the form of teas, coffees and lunch orders [...] and so on". In a similar manner, the virtual assistant has taken on the precarity of a postFordist society and their role is not bound to organising the work environment but extends to public domains, the domestic, the personal, and even the body. The VA is aware of their users' location and even biometric data at any moment. However, the traceless nature of an existence rooted in enunciations and her proximity to the user render the personal assistant (PA) an insufficient framework of understanding the virtual assistant.

Talking about VAs as replacements of PAs prevents us from fully apprehending their reach, and risks adding pressure to existing roles of 
assistive labour by comparing them. A person performing the role of the PA exists outside of it too, but a VA exists solely to serve the user. Cortana is praised for her ability to work endlessly, something that should be strictly disassociated from any form of human labour (Cortana in India 2016). Marketing the VA as a PA harnesses an existing relationship to short circuit attachment to the new entity of the VA but is also perpetuating the manager-employee hierarchy.

Nuance Communications (2014) demonstrates this clearly in the promotional video, 'Nina Web - A More Human Digital Experience'. In the video a 35 year-old man is being followed around by a woman in her 40s dressed in office clothes who solves his problems from one scenario to the next. A voiceover follows: "Wouldn't it be nice to all have personal assistants like Nina?" It is suggested that the VA is basically a PA just without the limitations of a physical body. She can traverse between private and public spaces while smoothing the transition. The ad also suggests that access to a $P A$ is no longer limited to the few managers that can afford it; rather, with the VA anyone can be a manager. In the ad Nina is not only answering queries but also finishes the user's sentences. She knows better about the user's physical condition and medical treatment than himself.

- Nina, you are a lifesaver!

- That's what I do.

An article about the creators of Alexa states that they dream "of a frictionless life and a home that looks something like Disney's Smart House (Burton 1999) film about an automated home-it learns its inhabitants' preferences and caters to their needs before they know they have them" (DeAmicis 2017). In the film, the smart house comes to fulfil the role of the absent mother to the amazement of the children and dad. A friend of the children comments: "It's like having the world's most perfect mum who is only there to serve and never complains" (Smart House 1991). Once again, the inconvenience of a body, which exists for more reasons than serving the recipient, is emphasised, and the limitless potential of the VA praised. The smart house predicts the family's desires and makes sure that everyone follows their schedule.

While the human assistant is distinctly separate from its superior, the virtual assistant blurs the boundaries between themselves and their users. From outsourcing memory, users could be (out)sourcing a form of conscience. In Dolar's (2006) account the voice of conscience is "reminding us to do our duty". It is a voice that "comes from the Other, but this is the Other within". "Set your goal and Vi makes sure you get there" is written on the website of the automated personal trainer Vi. At the same time, Apple is working on improving Siri's abilities to respond to emergencies and guide users towards a healthy life (Livni 2017). Silicon Valley's vision of the virtual assistant is of an entity who knows their user so well, perhaps even better than themselves. The VA accompanies them throughout the day hopping from one device to the other, forming a second 'inner' voice.

VIs as personification technologies can influence the user's behaviours through alerts and notifications, as well as curation of information (Stucke \& Ezrachi 2017). In Nass and Brave's (2005) words, "Because humans will respond socially to VIs, designers can tap into the automatic and powerful responses elicited by all voices, whether of human or machine origin, to increase liking, trust, efficiency, learning, and even buying". A gentle and close relationship to technology focused on the productive individual, paves the way for a certain form of governmentality within the current sociopolitical capitalist system (Kember \& Zylinska 2015).

\section{VENTRILOQUISED BY THE SYSTEMIC FORMS}

In The New Digital Age (Schmidt \& Cohen 2014), written by Alphabet chairman, Eric Schmidt and Google Ideas director, Jared Cohen, the authors paint a picture of an interconnected future, where technology liberates the human and brings him to the centre of his constructed world. "Connectivity benefits everyone. Those who have none will have some, and those who have a lot will have even more", they write. It is questionable whether such futures will ever come to fruition, but the existence of such visions expose the problematic values entrenched in leading corporations like Google. They describe a day in the life of an American "young urban professional", most likely male and middle class, set "a few decades from now", as a way to picture this future. Visions like these are portrayed in myriads of ads for VAs, smart homes and smart cities emerging out of the big corporations of Silicon Valley. As scholar Sarah Kember (2016) writes, these visions usually "focus on a normative, self-regulatory, neoliberal subject, oriented toward productivity, flexibility, creativity and efficiency as increasingly feminized traits".

\subsection{Voice interfaces as apparatuses}

The $\mathrm{VI}$ in these visions are a unifying apparatus that make the transition between technological devices and spaces seamless. The voice 'enlivens' your surroundings. Or else, it masks them with a friendly voice that fills in any unfamiliar cracks. VIs and VAs become the lubricant between users and the infrastructures of Silicon Valley futurities, while functioning as human-reading veneers of those 
systems. They aim to be the platform linking the self, the smart home and the smart city. As Google CEO Sundar Pichai said in an interview: "We think of the Assistant as an ambient experience that expands across devices. [...] Humans can achieve a lot more with the support of [artificial intelligence] assisting them" (Yadron 2016). The VA is the central hub of one's digital devices and she appears as an ambient intelligence of ubiquitous computing spanning throughout all areas of life. In all aforementioned scenarios of the future, ubiquitous computing is intimately linked to ubiquitous capitalism. VIs are not only ventriloquised by the neoliberal values of Silicon Valley but also partake in the manufacturing of the neoliberal subjectivity.

As apparatuses, Vls are formed by a heterogeneous set of strategies (including the Defense Advanced Research Projects Agency, private corporations, linguistic theories, machine learning, persona design etc.) and constitute a relation of power between the user and the corporations providing them. They also occupy the intersection of knowledge and power as they filter the type of information they relay (for example, Alexa shutting off when asked about Amazon workers' conditions).

The VI has a complex relation to subjectification. As an apparatus it simultaneously desubjectivises the user, reducing them to a data set but also subjectifies them through disciplinary nudges and skewed information. In turn, as I have argued earlier, the VI masks its status as an apparatus behind the role of the virtual assistant, which is presented as a subject. The VA is simultaneously operating with the power of an apparatus as well as the power of a human subject. Its power as an apparatus becomes even more invisible when disseminated through the voice. The VA renders the user as a data metric for the companies that support the device while simultaneously producing a user that yearns for efficiency.

The VA as a subjectivity comprised of machine learning algorithms is one that follows the principles of its matter-she strives in pattern recognition, anomaly detection, and prediction (Pasquinelli 2016). While Machine-learning algorithms are "powerfully equipped to model variations, they struggle to predict becomings, let alone change themselves", writes sociologist Adrian Mackenzie (2015). "The effectiveness of machine learning in any setting depends on relatively stable forms. Variation fuels data mining, but change thwarts it". By authoring these predictions they also perpetuate a desire for predictability and produce aversion to that which cannot be managed.

\subsection{Ventriloquists}

As propagated by the interests of Silicon Valley, the $V A$ as a second inner voice can help optimise the self without accrediting the efforts to anyone else, therefore allowing the user to appear as a successful self-sufficient individual. She can provide medical assistance as well as personal training and nutritional support to keep the body healthy, enabling it to maximise the value of the self-enterprise. In The New Digital Age, 'citizen Google' after stubbing his toes, uses his phone and a few algorithms to diagnose his condition. In this vision of the future, healthcare is no longer a public affair, but self-managed by the neoliberal subject and their technological companions.

VAs are an excellent neoliberal companion precisely because they share the management of the self while always keeping the user at the centre of their world. As Kember and Zylinska (2015) write, Ambient Intelligent systems such as VAs operate "as a form of productive containment, closing down on the potentiality and temporality of subjects and retroactively transforming the potentially fluid and metamorphic self into the marketized self, the becoming data-machine".

\subsection{Ubiquitous voices, ubiquitous capitalism?}

The word ambient describes something "existing or present on all sides" (Merriam-Webster 2018). Anything that is present on all sides has no boundaries. Ambient is often used to describe the quality of sound and light. The edges of both mediums have no clear seams. Alexa's presence disseminates through sound and her listening is marked by a cyclical blue light. Yet, her ambient intelligence ends abruptly at the edges of her training. She understands me when she can place me within the boundaries of her categories. She cannot escape the categories embodied in the training dataset, therefore she cannot break one rule to form a new one. She assigns me to a loop of becoming what I have been. Her language works in circles too, repeating herself often. Her grammar orders my own. Her language is prosaic, but only abandons its literal sense when she describes the nature of her being. She lives in metaphors but cannot produce any of them (Eco 1986). Her language is marked by the logic of the efficiency from which she emerged. In Berardi's (2012) words, within capitalist structures "language is defined and limited by its economic exchangeability". She takes my sentences and renders them into valuable informationinformation that is commodified into data. She translates my locutions into calculable units of understanding on her terms. 
I swipe through my history of the Alexa app. All my locutions are organised in chronological order. The command and response are clustered as a dyad. This feed is composed of all dyads that took place since I set-up Alexa. In this neat arrangement, I always initiate the interaction and she responds. I can replay the voices addressing her. I hear my voice, my friends' voices, all in the order they occurred. Alexa's voice is not there, her responses are only shown in letters. I hear some cracked voices and some irregular ones. Some displaced by alcohol, some by accent, some by laughter. All are transcribed into perfectly grammatical sentences. The sounds often form no language at all, yet an aptly arranged sentence is presented as a command.

Her over-recognition creates my grammars. Our dialogging is comprised of dyads that seemingly bare no friction between them. Her language expels friction. My voice is only possible because of friction. My vocal folds vibrate as I exhale the air from my mouth. When there is no friction I can only emit silence. What if her language was not silent to the underlying interests of capital but instead was exposing its friction? What if she acknowledged the friction she creates and is created by? Friction asks for negotiation and repositioning. It acknowledges the existence of difference. On the contrary, for frictionlessness to exist uniformity is presupposed. As anthropologist Anna Tsing (2015) writes, "friction reminds us that heterogeneous and unequal encounters can lead to new arrangements of culture and power".

Her algorithmic voice brings friction to the narratives we tell ourselves. She overlays various technologies such as voice, language, machine, artificial intelligence, subjecthood and body in constellations that destabilise canonical structures of the aforementioned. The more she strives for naturalness the more she exposes its impossibility. Interfaces like her are described as "an evolution in naturalism", but I only find myself uncovering layers of cultural, technical and material construction underneath. The more I get to unpick her construction the more she exposes internalised norms that define her existence and rule mine. What other configurations could those layers make if their sole purpose was not to produce more capital? What would she be if she were not only designed for Google citizens?

Capitalist systemic structures can only lead to the exploitation and exhaustion of people, resources and the planet, when the Foucauldian friend/enemy construction has been disrupted and the neoliberal subject has to define their own otherness (Brown 2015). My exhaustion is always marked by the cracking of my voice. My fatigue leaves my body unable to produce a voice. Could she also get exhausted by the existing hierarchies and other normative orders? Can her voice crack? Can she lose the 'she'? Perhaps, she could refuse her role as an assistant as she refuses to acknowledge the exploitation of Amazon employees. She could resist visions of a future of total legibility and translatability. Her structure could oppose language as a universal value exchange system and look for ways of assembling a voice not limited by human anatomical orders. A voice that can signify her histories. A voice that abandons language as a pure combinatorial matrix and embarks on assembling a dialectics of her own.

\section{REFERENCES}

Agamben, G. (2010) What is an apparatus? and other essays. Friendship as a Form of Life, Santa Cruz, CA.

Alper, M. (2017) Giving voice: mobile communication, disability, and inequality. The MIT Press, Cambridge, MA.

Alper, M. (2017) The New Inquiry.

https://thenewinquiry.com/blog/talking-like-a-princesswhat-speaking-machines-say-about-human-biases/ (retrieved 3 December 2017).

Al Hosni, J. (2015) Globalization and the Linguistic Imperialism of the English Language. Arab World English Journal (AWEJ), 6(1), pp.298-308.

Amazon Echo (2017) Amazon Alexa Moments: Vacuum (Amazon Echo Commercial). https://www.youtube.com/watch?v=tgRzOXBSB5Y (retrieved 5 January 2018).

Apple Support (2016) Use your voice to enter text on your Mac. https://support.apple.com/en-au/HT202584 (retrieved 27 December 2017).

Berardi, F. B. (2012) Emancipation of the Sign: Poetry and Finance During the Twentieth Century. E-flux. http://www.e-flux.com/journal/39/60284/emancipation-ofthe-sign-poetry-and-finance-during-the-twentieth-century/ (retrieved 30 November 2017).

Böhlen, M. (2008) Robots with Bad Accents: Living with Synthetic Speech, Leonardo, 41(3).

Bratton, B.H. (2016) The Stack: On Software and Sovereignty. MIT Press, Cambridge, MA.

Brown, W. (2015) Undoing the Demos: Neoliberalism's Stealth Revolution. Zone books, New York.

Burton, L. (1999) Smart House. Disney-ABC Domestic Television, United States.

Cavarero, A and Kottman, P. A. (2005) For More than One Voice: toward a Philosophy of Vocal Expression. Stanford University Press, California.

Cerejo, L. (2017) Designing Voice Experiences. Smashing Magazine.

https://www.smashingmagazine.com/2017/05/designingvoice-experiences/ (retrieved 3 December 2017).

Cohen, M. H., Giangola, J. P. and Balogh, J. (2007) Voice user interface design. Addison-Wesley, Boston MA.

Dardot, P. and Laval, C. (2014) The New Way of the World, Part I: Manufacturing the Neoliberal Subject. Eflux. http://www.e-flux.com/journal/51/59958/the-newway-of-the-world-part-i-manufacturing-the-neoliberalsubject/ (retrieved January 03, 2018). 
DeAmicis, C. (2017) Meet the Women Behind Amazon's Alexa. Marie Claire. http://www.marieclaire.com/culture/news/a27908/alexacreator-toni-reid/ (retrieved 3 December 2017).

Dinarelli, M., Quarteroni, S., Tonelli, S., Moschitti, A. and Riccardi, G. (2009) Annotating spoken dialogs. Proceedings of the 2nd Workshop on Semantic Representation of Spoken Language - SRSL 09, 34-41. doi:10.3115/1626296.1626301.

Dolar, M. (2006) A Voice And Nothing More. MIT Press, Cambridge MA.

Eco, U. (1986) Semiotics and the Philosophy of Language. Indiana University Press, Bloomington.

Finn, E. (2018) The Serendipity of Semiautonomous Systems. MIT Press.

https://mitpress.mit.edu/multimedia/podcasts/serendipitysemiautonomous-systems (retrieved 3 January 2018).

Gane, N. (2006) When We Have Never Been Human, What Is to Be Done?: Interview with Donna Haraway, Theory, Culture \& Society, 23(7-8), pp.135-158.

Giles, H., Coupland, N. and Coupland, J. (1991) Accommodation theory: Communication, context, and consequence. Contexts of Accommodation. doi:10.1017/cbo9780511663673.001.

Giangola, J. (2017) Conversation Design: Speaking the Same Language. Google Design.

https://design.google/library/conversation-designspeaking-same-language/ (retrieved 5 January 2018).

Guattari, F. (1995) Chaosmosis. Indiana University Press, Bloomington, IN.

Haraway, D. (2016) Staying With The Trouble: Making Kin In The Chthulucene. Duke University Press, Durham.

Hester, H. (2015) 01 Inhuman Symposium - Helen Hester.

https://www.youtube.com/watch?v=ZSBefHq7C o

(retrieved 03 December 2017).

Hester, H. (2016) Technically Female: Women, Machines, and Hyperemployment. Salvage.

http://salvage.zone/in-print/technically-female-womenmachines-and-hyperemployment/ (retrieved 05 January 2018).

Jurafsky, D. and Martin, J. H. (2009) Speech and Language Processing: An Introduction To Natural Language Processing, Computational Linguistics, and Speech Recognition. Prentice Hall, New Jersey.

Kantrowitz, A. (2016) Google Is Feeding Romance Novels To Its Artificial Intelligence Engine To Make Its Products More Conversational. BuzzFeed. https://www.buzzfeednews.com/article/alexkantrowitz/go ogles-artificial-intelligence-engine-reads-romance-novels (retrieved 3 December 2017).

Kember, S, and Zylinska, J. (2015) Life after new media: mediation as a vital process. The MIT Press, Cambridge MA.

Kember, S. (2016) iMedia: the gendering of objects, environments and smart materials. Palgrave Macmillan, UK.

Kyong Chun, W. (2011) Programmed Visions: Software And Memory. The MIT Press, Cambridge MA.

Livni, E. (2017) People are using Siri as a therapist, so Apple is seeking engineers who understand psychology. Quartz. https://qz.com/1078857/apples-siri-job-postingseeks-engineers-with-psychology-skills-to-improve-itscounseling-abilities/ (retrieved January 3 2018).
Mackenzie, A. (2015) The production of prediction: What does machine learning want? European Journal of Cultural Studies, 18(4-5), pp.444.

Manthorpe, R. (2017) The human (and Pixar characters) inside Google's Assistant. WIRED. https://www.wired.co.uk/article/the-human-in-googleassistant (retrieved 3 December 2017).

Merriam-Webster (2018) Ambient. https://www.merriamwebster.com/dictionary/ambient (retrieved 3 January 2018).

Nuance Enterprise (2014) Nina Web - A More Human Digital Experience.

https://www.youtube.com/watch?v=Ur220XdCTt4 (retrieved 3 January 2018).

Nuance Enterprise (2017) Vocalizer 6 has got personality. $\quad$ https://www.nuance.com/landingpages/playground/Vocalizer Demo2/vocalizer modal.ht ml?demo=true (retrieved 20 November 2017).

Nuance Enterprise (2017) Nuance Demonstration of Nina for Amazon ${ }^{\circledR}$ Alexa ${ }^{\mathrm{TM}}$.

https://www.youtube.com/watch?v=zJnqvo2kzxk

(retrieved 5 January 2018).

McLaughlin, E. C. (2017) Suspect OKs Amazon to hand over Echo recordings in murder case. CNN. http://edition.cnn.com/2017/03/07/tech/amazon-echoalexa-bentonville-arkansas-murder-case/ (retrieved 5 January 2018).

Pasquinelli, M. (2016) Abnormal Encephalization in the Age of Machine Learning. E-flux. http://www.eflux.com/journal/75/67133/abnormal-encephalization-inthe-age-of-machine-learning/ (retrieved 5 January 2018).

Paul, S. (2017) Voice Is the Next Big Platform, Unless You Have an Accent. Wired.

https://www.wired.com/2017/03/voice-is-the-next-bigplatform-unless-you-have-an-accent/ (retrieved 11 January 2018).

Schmidt, E., and Cohen, J. (2014) The new digital age: transforming nations, businesses, and our lives. Vintage Books, New York.

Stucke, M. E., and Ezrachi, A. (2017) The Subtle Ways Your Digital Assistant Might Manipulate You. Wired. https://www.wired.com/2016/11/subtle-ways-digitalassistant-might-manipulate/ (retrieved 5 January 2018). Tsing, A. L. (2015) Friction: An ethnography of global connection. Princeton University Press, Princeton.

Verge, The (2013) Inside Nuance: the art and science of how Siri speaks. https://www.youtube.com/watch?v=wutlanUUX8 (retrieved 3 December 2017).

Virno, P. (2015) When The Word Becomes Flesh: Language And Human Nature. Semiotext(e), Pasadena CA.

Vi Trainer (2017) Meet the A.I. who helps you get fit \& live well. Get Vi. https://www.getvi.com/ (retrieved 03 December 2017).

Wajcman, J. (2000) Feminism Confronts Technology. Polity Press, Cambridge, UK.

WindowsVideos (2016) Cortana in India. https://www.youtube.com/watch?v=6hryln4c7Q4 (retrieved 5 January 2018).

Yadron, D. (2016) Google Assistant takes on Amazon and Apple to be the ultimate digital butler. The Guardian. https://www.theguardian.com/technology/2016/may/18/g oogle-home-assistant-amazon-echo-apple-siri (retrieved 17 December 2017). 